\title{
Medizinsysteme - Begriffsverwirrungen - Patientenverdummung
}

A uf der Jahrestagung des Kuratoriums der Akademie für Wissenschaft, Wirtschaft und Technik an der Universität Ulm präsentierte Professor J. Aschoff (Neurologe und Psychiater) neulich einen spektakulären Vortrag zum Thema „Medizinsysteme - Begriffsverwirrungen - Patientenverdummung“. Er griff dabei das Problem der nicht selten gleichnamigen aber doch meist inhaltlich unterschiedlich definierten Begriffe in unterschiedlichen Medizinsystemen auf.

Verschärft wird dieses Problem durch eine häufig unverständlich schlampige, aber durch den interdisziplinären Charakter der Medizin bedingte Nutzung von Begriffen. Beispielhaft möchte ich hier einige Teilbereiche herausgreifen. So besteht keineswegs ein einheitlicher Wissensstand über Begriffe wie alternative Medizin, traditionell chinesische oder tibetische und ayurveda-medizinische Systeme. Sie werden nebeneinander nicht nur benannt, sondern auch angewendet - obwohl das Verständnis über deren Inhalt selten auf profunden und umfassenden Studien beruht. Ihre Grundlage sind eher anwendungsorientierte Schulungsergebnisse mit starken Glaubensüberzeugungen und emotionaler Übertünchung.

Aber schon in der Definition dessen, was wir unter Schulmedizin verstehen, besteht große Unkenntnis. Nicht selten wird die Schulmedizin gleichgesetzt mit „westlicher Medizin“ oder ganz negativ mit „Apparatemedizin“. Es ist jedoch ethisch höchst bedenklich, wenn mit diesen häufig als Abschreckung benutzten Begriffen Patienten in ihrer persönlichen Not so verunsichert werden, dass sie bei entsprechender Überredungskunst doch sehr leicht Opfer auch von Scharlatanen werden. Man braucht gar nicht so kompromisslos vorzugehen und nun für jede Handlung am Patienten evidenzbasierte Untersuchungsstudien zu fordern.

Dennoch muss man nachfragen, mit welchem Recht eine einseitig weltanschaulich geprägte Begriffsmissbräuchlichkeit zum Beispiel Therapien nutzt, die auf zugegeben charismatische Personen zurückgehen, die sich aber mit der Medizin als Heilkunde nicht wirklich befasst haben. Wir sollten uns daher bei der Verwendung von Begriffen die Mühe machen, über deren Inhalte sehr genau nachzudenken, weil ohne diese rationale Präzisierung die Verantwortlichkeit gegenüber dem Patienten stark eingeschränkt wird. So ist unfassbar, mit welcher Bandbreite Begriffe im Gebrauch sind, wenn man alleine im Bereich der psychiatrischen Medizin die Palette der Berufs- und Fachbezeichnungen vom Psychotherapeuten bis zum Neuropsychiater oder Neurobiologen und damit den Erfindungsreichtum bewundert, der selten die Abgrenzung oder gar die präzisen Inhalte dieser Begriffe erkennen lässt.
Diese Nachlässigkeitssünden, die häufig die weltanschaulich fixierten Einseitigkeiten übertünchen, finden sich bis hin zu einer Unzahl falscher Einzelbegriffe: Als Beispiel sei nur daran erinnert, wie undurchdacht auch in bekannten und großen Lehrbüchern der Flüssigkeitsstatus eines Menschen und seine Therapie mit Hydrierung beschrieben wird. Definitionsgemäß ist „Hydrierung“ jedoch der chemische Prozess der Wasserstoffanlagerung unter Anwendung von Nickel als Katalysator. Der einzig richtige Ausdruck für den Flüssigkeitszustand eines Patienten ist die Hydratation bzw. der Hydratationsstatus.

Wir sind der Meinung, dass sowohl die Begriffe als auch die als ärztliche Heilmethoden eingesetzten Verfahren nur dann nicht zur Patientenverdummung führen, wenn sich der Arzt in seiner verpflichtenden verantwortungsvollen Handlung darüber im klaren ist, dass er das, was er tut, wirklich verstanden hat und verantwortlich anwendet. Wir hoffen, dass auf dieser Verständnisebene der Leser des klinikarzt eine umso bessere Motivation gewinnt, da wir die Sicherheit für die Begriffsanwendungen in den einzelnen Arbeiten auf einem hohen Niveau sicherstellen wollen.

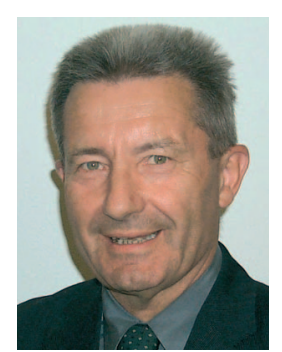

Prof. Dr. Dr. Dr. A. Grünert, Ulm

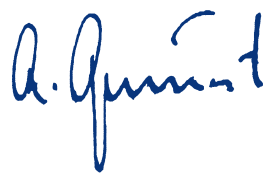

